(RC) in test counts increased (RC 1.22; 95\% CI 1.14 to 1.30) after campaigns, but no significant increase was seen for number of positive tests (RC $1.10 ; 95 \%$ CI 0.93 to 1.30 ) nor for test positivity rate (RC 0.93 ; $95 \%$ CI 0.81 to 1.07). Heterogeneity between studies was very high for all analyses. It was not possible to explore outcomes by type of campaign components. Seven major qualitative themes were identified: targeting of campaigns; quality of materials and message; language; anonymity; use of technology; relevance; and variety of testing options.

Conclusions Health promotion campaigns aiming to increase chlamydia testing in those aged 15-24 may show some effectiveness in increasing overall numbers of tests, however numbers of positive tests do not follow the same trend.

\section{P216 UMBRELLA COMBINED SEXUAL HEALTH SERVICES, CHLAMYDIA SCREENING AND DEPRIVATION: ARE WE ACHIEVING CONTINUED SCREENING OF HIGH-RISK POPULATIONS?}

${ }^{1} \mathrm{E}$ Pearce*, ${ }^{1} \mathrm{~K}$ Jolly, ${ }^{1} \mathrm{M}$ Price, ${ }^{2} \mathrm{H}$ Mohammed, ${ }^{2} \mathrm{H}$ Allen, ${ }^{3} \mathrm{~J}$ Ross. ${ }^{1}$ University of Birmingham, Birmingham, UK; ${ }^{2} H I V$ and STI Dept, National Infection Service, Public Health UK; ${ }^{3}$ University Hospitals Birmingham, Birmingham, UK

\subsection{6/sextrans-2021-sti.303}

Introduction Combined sexual health services have been provided in Birmingham and Solihull by 'Umbrella' since 2015. The local population is younger and more deprived than the average English sexual health service population. We investigated if the 2015 Umbrella redesign, and subsequent health promotion campaigns, affected uptake of chlamydia screening in those aged 15-24 in the most deprived groups compared to the total population.

Methods Weekly aggregated data for gender, socioeconomic status (IMD-5) and chlamydia test status with test result were obtained from Public Health England's STI and HIV Surveillance Team for the period 2012 - 2018. Overall percent positivity and yearly moving averages for tests, positive tests and percent positivity, by gender, were calculated by the study team for total population, and the most deprived. Number of positive tests and percent positivity were graphically represented and mapped against dates of service redesign and subsequent campaigns. Data analysis was completed using Microsoft Excel.

Results Positive tests in all females decreased gradually between late 2014 and early 2016 before recovering partially in 2017: this effect was not replicated for all other groups, and neither Umbrella redesign nor health promotion campaigns showed any effect on absolute numbers. In females, percent positivity increased steadily during the study period, from $10 \%$ to $12 \%$ per week in the most deprived females and $8 \%$ to $10 \%$ per week in all females. Percent positivity showed a clear link with service redesign in males: in the year following redesign, rates in all males rose from $8 \%$ to $15 \%$ per week, and from $10 \%$ to $18 \%$ per week in the most deprived males.

Conclusion Umbrella redesign had a significant effect on detection of chlamydia cases in males; this effect is less obvious in females, and appears not to relate to the promotional campaigns. Deprived populations appear well served by Umbrella.

\section{P217 THE IMPACT OF UREAPLASMA INFECTIONS ON PREGNANCY COMPLICATIONS}

${ }^{1} \mathrm{~A}$ Bartkeviciute*, ${ }^{2} \mathrm{D}$ Bartkevičienè. ${ }^{1}$ Clinic of Dermatovenereology, Institute of Clinical Medicine, Faculty of Medicine, Vilnius University, Lithuania; ${ }^{2}$ Centre of Obstetrics and Gynecology, Institute of Clinical Medicine, Faculty of Medicine, Vilnius University, Lithuania

\subsection{6/sextrans-2021-sti.304}

The aim of this study was to assess if ureaplasmas are associated with pregnancy complications and diseases in newborns. Pregnant women with complaints and threatening signs of preterm delivery were included. A sample, taken from the endocervical canal and from the surface of the cervical portion, was sent to the local microbiology laboratory for DNA detection of seven pathogens: Chlamydia trachomatis, Mycoplasma hominis, Mycoplasma genitalium, Ureaplasma parvum, Ureaplasma urealyticum, Neisseria gonorrhoeae, and Trichomonas vaginalis. The Pearson Chi-Square test was used to determine the difference in unpaired categorical data. A two-sided $p$ value $<0.05$ was considered to be statistically significant. In all, 50 pregnant women with complaints and threatening signs of preterm delivery were included. Premature rupture of uterine membranes was found in $23(46 \%)$ of the patients and 38 women $(76 \%)$ had preterm delivery. Ureaplasma infections were associated with a premature rupture of membranes $(\mathrm{p}<$ $0.004)$, the placental inflammation ( $p<0.025$ ), a newborn respiratory distress syndrome $(p<0.019)$. Ureaplasmas could have affected the preterm leakage of fetal amniotic fluid and are associated with the placental inflammation and a newborn respiratory distress syndrome.

\section{P218 MYCOPLASMA GENITALIUM: THE MOST PREVALENT STI IN SASKATCHEWAN, CANADA, HAS A HIGH PREVALENCE OF RESISTANCE TO MACROLIDES AND FLUOROQUINOLONES}

${ }^{1,2}{ }^{2}$ Gohil ${ }^{*},{ }^{3} \mathrm{~L}$ Mushanski, ${ }^{2} \mathrm{~T}$ Wanlin, ${ }^{1,2} \mathrm{~A}$ Lepe, ${ }^{3} \mathrm{~A}$ Lang, ${ }^{3} \mathrm{~J}$ Minion, ${ }^{1,2}{ }^{2}$ Dillon. ${ }^{1}$ University of Saskatchewan, Saskatoon, Canada; ${ }^{2}$ Vaccine and Infectious Disease Organization, Saskatoon, Canada; ${ }^{3}$ Saskatchewan Health Authority, Roy Romanow Provincial Laboratory, Regina, Canada

\subsection{6/sextrans-2021-sti.305}

Objectives Mycoplasma genitalium, a sexually transmitted infection (STI), is one of the most common causes of nongonococcal urethritis worldwide. An increase in resistance to antibiotics (i.e., azithromycin and moxifloxacin), recommended for treating M. genitalium infections has been observed. We describe the prevalence of $\mathrm{M}$. genitalium and its antimicrobial resistance (AMR) in Saskatchewan (SK), Canada.

Methods M. genitalium was identified using the Aptima Mycoplasma genitalium Assay (MG-TMA) on 1977 specimens collected (January and March/April 2019) for the diagnosis of Chlamydia trachomatis and Neisseria gonorrhoeae. Mutations implicated in AMR were ascertained using PCR and DNA sequencing of $23 \mathrm{~S}$ rRNA (azithromycin) and parC (moxifloxacin) from MG-TMA positive specimens.

Results The prevalence of $\mathrm{M}$. genitalium in SK was 9.6\% $(189 / 1977)$ and was higher than the prevalence of N. gonorrhoeae $(3.09 \%)$ and C. trachomatis $(6.85 \%)$ during the same time. Mutations mediating macrolide resistance (positions $2058 / 2059$ in $23 \mathrm{~S}$ rRNA) were observed in $63.6 \%(70 / 110)$ 
of the specimens, while resistance to moxifloxacin (S83I in ParC) was observed in $10.6 \%(9 / 85)$ of the specimens tested. Mutations predictive of dual resistance (both $23 \mathrm{~S}$ rRNA and ParC) were observed in $2.12 \%(4 / 189)$ of the specimens. Females 20-24 years had the highest prevalence $(18.3 \%$, $\mathrm{p}<0.001)$ of infection. In females, M. genitalium was significantly associated with C. trachomatis or N. gonorrhoeae/C. trachomatis $(p<0.001)$ co-infection. The prevalence of $M$. genitalium in a northern region was statistically significantly higher $(p=0.012)$, as compared to other regions in the province.

Conclusions The prevalence of M. genitalium (9.6\%) and associated resistance to azithromycin (63.6\%) in Saskatchewan is high, suggesting that empiric azithromycin therapy may not be adequate for treating these infections. A potential risk of azithromycin treatment failure could be avoided by performing resistance-guided therapy, as azithromycin is also a recommended drug for the treatment of $\mathrm{N}$. gonorrhoeae (combined with ceftriaxone) and C. trachomatis infections in Canada.

\section{P219 POLICY AND PROGRAM RECOMMENDATIONS FOR STBBI INCENTIVE-BASED TESTING IN HIGH-INCOME COUNTRIES: A SYSTEMATIC REVIEW}

1,2 J Finlay*, IJ Krahn, ${ }^{2} \mathrm{~T}$ Lambert, ${ }^{2} \mathrm{G}$ Meyer, ${ }^{2,3} \mathrm{~A}$ Singh, ${ }^{3} \mathrm{~V}$ Caine. ${ }^{1}$ University of Calgary, Calgary, Canada; ${ }^{2}$ Alberta Health Services, Edmonton, Canada; ${ }^{3}$ University of Alberta, Edmonton, Canada

\subsection{6/sextrans-2021-sti.306}

Background Despite increasing access to treatment and testing, rates of sexually transmitted and blood-borne infections (STBBI) continue to rise. At the same time, screening uptake remains suboptimal. Incentives (e.g. cash, gift cards, prize draws) have been proposed as a way to increase the immediate rewards of STBBI testing and facilitate a greater uptake of screening. This systematic review was conducted to determine if patient incentives increase STBBI screening uptake in highincome countries.

Methods Our review was performed according to the recommendations of the Preferred Reporting Items for Systematic Reviews and Meta-Analyses (PRISMA) statement and the Cochrane Handbook for Systematic Reviews of Interventions. MEDLINE, EMBASE, PsycINFO, CINAHL, Scopus, and Cochrane Library were searched from 2013 to 2020. Inclusion criteria were: English language, high-income countries, primary research studies, and age $>13$ years. Study quality was assessed using Joanna Briggs Institute quality assessment tools.

Results The search yielded 6217 abstracts.13 studies met the inclusion criteria. Of these studies, five were located in the United States, three in the United Kingdom, and five in Australia. Seven studies took place in the community, five studies took place in clinical settings, and one study utilized a combination of community and clinical settings. Study design and intervention heterogeneity precluded meta-analysis or data pooling. Populations screened included: post-secondary students, parolees or probationers, youth, and inner-city emergency department patients. Incentivized STBBI tested were HIV $(n=5)$, chlamydia $(n=7)$, and multiple infections $(n=1)$. Incentives offered were monetary (cash/gift cards/not specified) $(n=8)$, non-monetary $(n=2)$, and mixed $(n=3)$.

Conclusion Both monetary and non-monetary incentives enhance STBBI screening in high-income countries.
Incentivized testing programs are most effective when developed specific to context and target population. Further research is needed to analyze incentivized screening across similar study designs and to evaluate long-term effectiveness.

\section{P220 THE BURDEN OF HIV ON MALARIA AND SEXUALLY TRANSMITTED AND REPRODUCTIVE TRACT INFECTIONS AMONG PREGNANT WOMEN OF RURAL, ZAMBIA}

${ }^{1} E$ Chaponda*, ${ }^{2} \mathrm{R}$ Chico, ${ }^{2} \mathrm{~J}$ Bruce, ${ }^{1} \mathrm{C}$ Michelo, ${ }^{2} \mathrm{D}$ Chandramohan. 'University of Zambia, Lusaka, Zambia; ${ }^{2}$ London School of Hygiene and Tropical Medicine, London, UK

\subsection{6/sextrans-2021-sti.307}

Background Malaria and curable sexually transmitted and reproductive tract infections (STIs/RTIs) are important causes of adverse birth outcomes. HIV has been associated with STIs/ RTIs and malaria infection. The study objectives were to estimate the prevalence of malaria and STIs/RTIs and to identify risk factors for infection in rural Zambia.

Methods A total of 1,086 pregnant women were enrolled at antenatal care booking, socio-demographic information and biological samples were collected for diagnosis of malaria, STIs/RTIs and routine HIV testing.

Results Participants had a mean age of 25.6 years. The prevalence of HIV was $13.2 \%$ (95\% confidence interval [CI], 11.3, 15.3 ) and the highest burden was in multigravidae (15.9\%) followed by secundigravidae $(13.9 \%)$ and was lowest in primigravidae $(8.0 \%), \mathrm{P}=0.018$. Of 1084 women, 700 had at least one STI/RTI (95\% CI, 64.6\%; 95\%, 61.7, 67.4). The prevalence of STIs/RTIs was as follows: chlamydia $5.2 \%$, (95\% CI, 4.0, 6.6); gonorrhoea 3.1\% (95\% CI, 2.2, 4.4); trichomoniasis $24.8 \%$ (95\% CI, 22.3, 27.5); bacterial vaginosis 48.7\% (95\% CI, 45.2, 51.2) and syphilis 7.1\% (95\% CI, 5.6, 8.7). The malaria prevalence measured by PCR was $57.8 \%$ (95\% CI, 54.9, 60.8).

HIV infection was associated with having gonorrhoea, (aOR $=2.80 ; 95 \%$ CI: 1.25, 6.26); bacterial vaginosis $(\mathrm{aOR}=$ 2.41; 95\% CI: 1.66, 3.51); syphilis infection $(\mathrm{aOR}=2.56$; 95\% CI: 1.48, 4.41); having any STIs/RTIs $(\mathrm{aOR}=2.10$; $95 \% \mathrm{CI}: 1.39,3.19)$ and malaria infection $(\mathrm{aOR}=1.46$; 95\% CI 1.00-2.13, $\mathrm{P}=0.045)$.

Conclusion HIV infection plays an important role in the prevalence of malaria and STIs/RTIs although it's effect on malaria prevalence in this population was neutralised by its concentration in multigravidae who have acquired immunity against malaria. Preventive and control efforts against malaria and STIs/RTIs should be targeted at the groups that needs them most in resource limited settings.

\section{P221 SYNDROMIC MANAGEMENT OF CURABLE SEXUALLY TRANSMITTED AND REPRODUCTIVE TRACT INFECTIONS AMONG PREGNANT WOMEN IN RURAL ZAMBIA}

${ }^{1} E$ Chaponda*, ${ }^{2} \mathrm{R}$ Chico, ${ }^{2} \mathrm{~J}$ Bruce, ${ }^{1} \mathrm{C}$ Michelo, ${ }^{2} \mathrm{D}$ Chandramohan. ${ }^{1}$ University of Zambia, Lusaka, Zambia; ${ }^{2}$ London School of Hygiene and Tropical Medicine, London, UK

\subsection{6/sextrans-2021-sti.308}

Background This study estimated the prevalence of curable sexually transmitted and reproductive tract infections (STIs/ RTIs) among pregnant women attending antenatal care (ANC) in rural Zambia and evaluated the effectiveness of syndromic 